\title{
Perfil de Acadêmicos de Odontologia sobre Biossegurança
}

\author{
Profile of Dental Students about Biosafety
}

Juan Érico de Xerez ${ }^{1}$, Hugo Costa Neto ${ }^{1}$, Francisco Lopes da Silva Júnior ${ }^{2}$, Conceição Aparecida Dorneles Monteiro Maia ${ }^{3}$, Hébel

Cavalcanti Galvão ${ }^{4}$, Manuel Antonio Gordón-Núñez ${ }^{5}$

\begin{abstract}
Aim: The professional formation in Dentistry requires preparation and knowledge regarding the use of universal biosafety measures against exposure to infectious agents. This descriptive study aimed to evaluate the level of knowledge of dentistry students about biosafety, and identify the most frequent doubt about preventive measures in dental care environment, also assess the student's interest by biosafety. Material and methods: The sample consisted of 358 students from three universities. A questionnaire with subjective and objective questions was applied to students at 1st, 5th and last periods. Results: It was observed that $92.6 \%$ of students knew the meaning of biosafety and $92.3 \%$ reported the use of PPE for their dentist and for the patient. When asked if they would know to take care in clinical environment, $291(82.7 \%)$ students answered that they would know how to care and $59(16.8 \%)$ not, $94.9 \%$ of the latter were students of the first period, in this group, $38.4 \%$ of students responded not be important to consider the patient as a potential transmitter of disease. In the total sample, $98.6 \%$ considered to be important the topic of the research. Conclusions: It was concluded that it is important introduce basic content on biosafety in the pre-clinical periods, aiming at the entry of students to the clinical activities more safely. It is emphasized the importance of supervision and periodic update of practices and knowledge about preventive measures facilitating the exercise of academic activities in safety at all course periods
\end{abstract}

Key words: Dentistry. Biosafety. Infection control. Students.

\section{Resumo}

Objetivo: A formação do profissional da Odontologia requer preparo e esclarecimento quanto ao uso das medidas universais de biossegurança em virtude da exposição a agentes infectocontagiosos. Este estudo descritivo teve como objetivo avaliar o nível de conhecimento de uma população de acadêmicos de Odontologia a respeito de biossegurança, além de identificar as dúvidas mais frequentes sobre medidas preventivas no ambiente odontológico e avaliar 0 interesse desses acadêmicos em relação ao tema biossegurança. Materiais e métodos: A amostra foi constituída por 358 alunos de três universidades. Foi aplicado um questionário contendo perguntas objetivas e subjetivas em alunos dos $1^{\circ}, 5^{\circ} \mathrm{e}$ últimos períodos. Resultados: Observou-se que $92.6 \%$ dos alunos conheciam o significado de biossegurança e $92.3 \%$ relatou o uso de EPI pelo seu CD e para o paciente. Frente a um atendimento na clínica, $291(82.7 \%)$ alunos responderam que saberiam se cuidar e 59 (16.8\%) que não, sendo $94.9 \%$ destes últimos, alunos do primeiro período, neste grupo, $38.4 \%$ responderam não ser importante considerar o paciente como potencial portador de doença. Conclusões: Conclui-se que, é importante ministrar conteúdos básicos sobre biossegurança nos períodos pré-clínicos, visando a entrada dos alunos às atividades clínicas com maior segurança. Ressalta-se ainda a importância da fiscalização e atualização periódicas de práticas, e conhecimentos das medidas preventivas adequadas que permitam o exercício de atividades acadêmicas em segurança em todos os períodos do curso.

Palavras chave: Odontologia. Biossegurança. Controle de infecções. Estudantes.
${ }^{1}$ Acadêmico do Curso de Odontologia, Universidade Federal do Rio Grande do Norte, Natal/RN, Brasil.

${ }^{2}$ Aluno do Curso de Mestrado em Odontologia do Programa de Pós-Graduação em Saúde Coletiva da UFRN, Natal - RN, Brasil.

${ }^{3}$ Profa, Universidade Potiguar, M.D, Doutoranda do Programa de Pós-Graduação em Patologia Oral da UFRN, Natal - RN, Brasil.

${ }^{4}$ Profa, M.D, Ph.D em Patologia Oral, Professora Associada III, Disciplina de Patologia Oral, Departamento de Odontologia, Universidade Federal do Rio Grande do Norte, Natal - RN, Brasil.

${ }^{5}$ Prof, M.D, Ph.D em Patologia Oral, Professor Efetivo de Processos Patológicos, Curso de Odontologia, Universidade Estadual da Paraíba - Campus VIII, Araruna PB, Brasil.

Correspondência: Manuel Antonio Gordón-Núñez

Endereço: Universidade Federal do Rio Grande do Norte (UFRN)

Av. Senador Salgado Filho, 1787 - Lagoa Nova, CEP 59056-000 - Natal - RN, Brasil

Fone: (84) 32154108

E-mail: gordonnunez28@yahoo.com / hebel.galvao@yahoo.com.br

Data de Submissão: 07/08/2012

Data de Aceite: 14/08/2012

\section{Introdução}

A biossegurança é o conjunto de ações voltadas para a prevenção, minimização ou eliminação de riscos que podem comprometer a saúde do homem, dos animais, do meio ambiente ou a qualidade dos trabalhos (PENNA et al., 2010).

Os acidentes de trabalho são bastante frequentes em Odontologia, estando associados, usualmente, à manipulação constante de objetos pontiagudos e motores de alta rotação, os quais podem causar ferimentos e abrasões nos profissionais e/ou nos pacientes, sendo as agulhas os objetos mais frequentemente envolvidos nestes (SILVA, G. et al., 2012). Os riscos de exposição ao HIV (Vírus da Imunodeficiência Humana), HBV (Vírus da Hepatite B) e HCV (Vírus da Hepatite C) são proporcionais ao manuseio de objetos perfurocortantes e fluidos orgânicos (SILVA, G. et al., 2012). Além disso, fragmentos dentários ou líquidos podem ser projetados com consequente risco de lesão cutânea ou ocular (SANTOS; PELLOGIA, 2002).

A equipe odontológica está sob constante risco de contaminação por agentes infecciosos. Com o surgimento da infecção pelo HIV, foi que os acidentes ocupacionais, principalmente os pérfuro-cortantes, começaram a ter maior atenção por parte dos profissionais e pesquisadores; trazendo à tona a necessidade de maiores cuidados com a prevenção de acidentes relacionados ao contato com materiais potencialmente contaminantes como o sangue (SILVA, G. et al., 2012; BROZOSKI et al., 2010).

Estudos sobre a aderência de acadêmicos de Odontologia às normas de controle de infecções têm mostrado que embora a 
maioria dos mesmos receba capacitação teórico-prática sobre biossegurança, sendo cobrados constantemente no tocante à realização de procedimentos de controle de infecções por parte das equipes docentes, ainda persistem falhas e falta de conhecimento (MEDEIROS; CARDOSO; FERREIRA, 1998; SILVA, R. et al., 2007).

Baseado no antes exposto, este estudo avaliou o nível de conhecimento de um grupo de acadêmicos de Odontologia a respeito de biossegurança, além de identificar as dúvidas mais frequentes sobre o tema.

\section{Materiais e Métodos}

Este estudo descritivo foi aprovado pelo Comitê de Ética em Pesquisa da UFRN. Os alunos foram informados sobre o objetivo e caráter do estudo, sendo convidados a participar voluntariamente mediante a assinatura do Termo de Consentimento Livre e Esclarecido, e seguidamente preenchimento de um questionário estruturado (adaptado de Passos et al) com 08 questões objetivas e subjetivas sobre biossegurança.

A população do estudo foi composta por alunos matriculados no primeiro, quinto e últimos períodos curriculares da Universidade Federal do Rio Grande do Norte (UFRN), da Universidade Estadual do Rio Grande do Norte (UERN) e da Universidade Potiguar (UnP).

Os dados foram processados e submetidos à análise estatística descritiva utilizando-se o software estatístico SPSS ${ }^{\odot}$ (Statistical Package for the Social Sciences, 17.0, Chicago, IL, USA).

\section{Resultados}

Participaram do estudo 358 alunos, sendo 95 (26.5\%) da UFRN, 205 (57.3\%) alunos da UnP e 58 (16.2\%) da UERN, cuja distribuição por período letivo está representada na Figura 1.

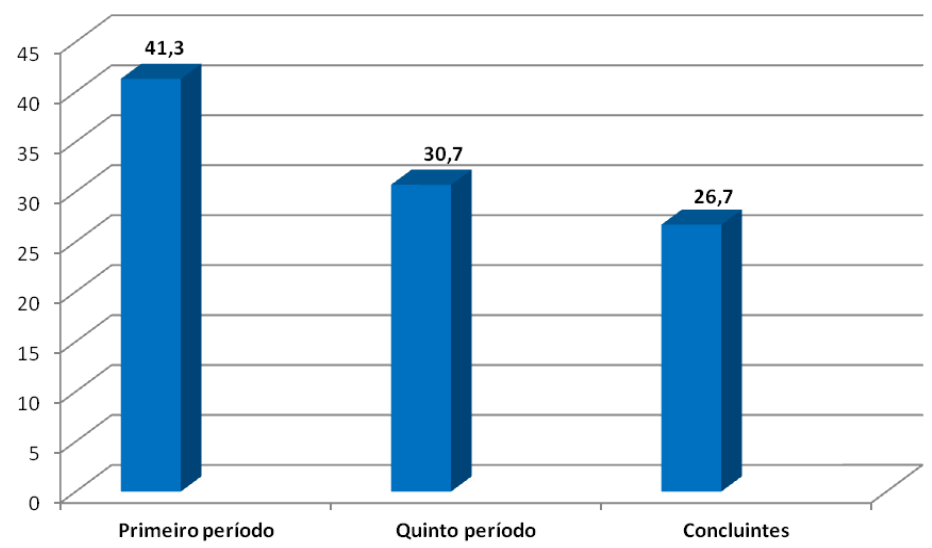

Figura 1. Distribuição da amostra considerando os períodos letivos avaliados. Natal - RN, 2012.

A maioria da amostra (93.3\%) afirmou ter conhecimento sobre o significado de biossegurança e $6.7 \%$ não conhecia o significado (Tabela 1).

Quando questionados se já haviam assistido alguma palestra ou aula sobre o tema de biossegurança, $249(69.6 \%)$ alunos responderam que sim e $109(30.4 \%)$ (Tabela 1$)$.

No quesito em que os alunos eram questionados se já haviam notado se seus dentistas utilizavam algum tipo de equipamento de proteção individual (EPI) para o $C D$ e para o paciente, 331 (92.5\%) alunos responderam que sim e 27 (7.5\%) alunos responderam que não haviam notado nenhum tipo de EPI. Dentre os alunos que responderam ter notado o uso de algum tipo de EPI, $320(9.4 \%)$ citaram quais eram esses equipamentos, verificandose que $64(17.9 \%)$ relataram o uso de EPI completo (touca, máscara, óculos, luvas, jaleco e sapato fechado) por parte dos CDs (Figura 2).

Tabela 1. Valores numéricos e percentuais das respostas dadas às perguntas dicotômicas do questionário sobre biossegurança. Natal $\mathrm{RN}, 2012$

\begin{tabular}{|c|c|c|c|c|c|c|}
\hline & $1^{\circ} p$ & íodo & $5^{\circ}$ perí & do & Conclu & tes \\
\hline & Sim & Não & Sim & Não & Sim & Não \\
\hline $\begin{array}{l}\text { Conhece o } \\
\text { significado de } \\
\text { biossegurança. }\end{array}$ & $124(83.8)$ & $24(16.2)$ & $110(100)$ & $0(0.0)$ & $100(100)$ & $0(0.0)$ \\
\hline $\begin{array}{l}\text { Palestra/aula } \\
\text { sobre } \\
\text { biossegurança. }\end{array}$ & $48(32.4)$ & $100(67.6)$ & $104(94.5)$ & $6(5.5)$ & 97 (97) & $3(3.0)$ \\
\hline $\begin{array}{l}\text { Uso de EPI para } \\
\text { CD e paciente. }\end{array}$ & $132(89.2)$ & $16(10.8)$ & $10(96.4)$ & $4(3.6)$ & $93(93)$ & $7(7.0)$ \\
\hline $\begin{array}{l}\text { Saberia } \\
\text { exatamente como } \\
\text { se cuidar ao } \\
\text { atender } \\
\text { pacientes. }\end{array}$ & $90(60.8)$ & $58(39.2)$ & 109 (99.1) & $1(0.9)$ & $98(98)$ & $2(2.0)$ \\
\hline $\begin{array}{l}\text { Considerar todo } \\
\text { paciente como } \\
\text { potencial portador } \\
\text { de alguma } \\
\text { doença. }\end{array}$ & $90(60.8)$ & $58(39.2)$ & $101(91.8)$ & $9(8.2)$ & $97(97)$ & $3(3.0)$ \\
\hline $\begin{array}{l}\text { Considera } \\
\text { importante o tema } \\
\text { "biossegurança" } \\
\text { para o recém- } \\
\text { ingresso ao } \\
\text { Curso de } \\
\text { Odontologia. }\end{array}$ & $145(98)$ & $3(2.0)$ & $110(100)$ & $0(0.0)$ & $100(100)$ & $0(0.0)$ \\
\hline
\end{tabular}

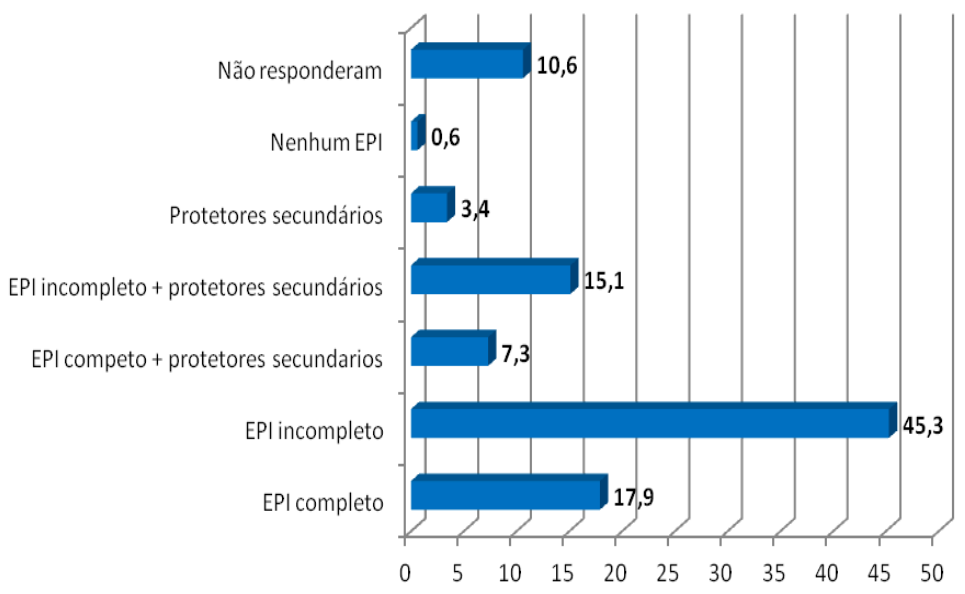

Figura 2. Distribuição da amostra em relação ao relato de uso de EPI pelo cirurgião-dentista e para o paciente. Natal - RN, 2012. 
À pergunta "Se você fosse atender em uma das clinicas de sua faculdade, você saberia como, exatamente, se cuidar?", 297 $(83 \%)$ alunos responderam que sim e $61(17 \%)$ que não saberiam (Tabela 1).

Quando questionados sobre quais eram as dúvidas em relação à biossegurança em ambiente odontológico, 259 (72.3\%) alunos responderam (Figura 3).

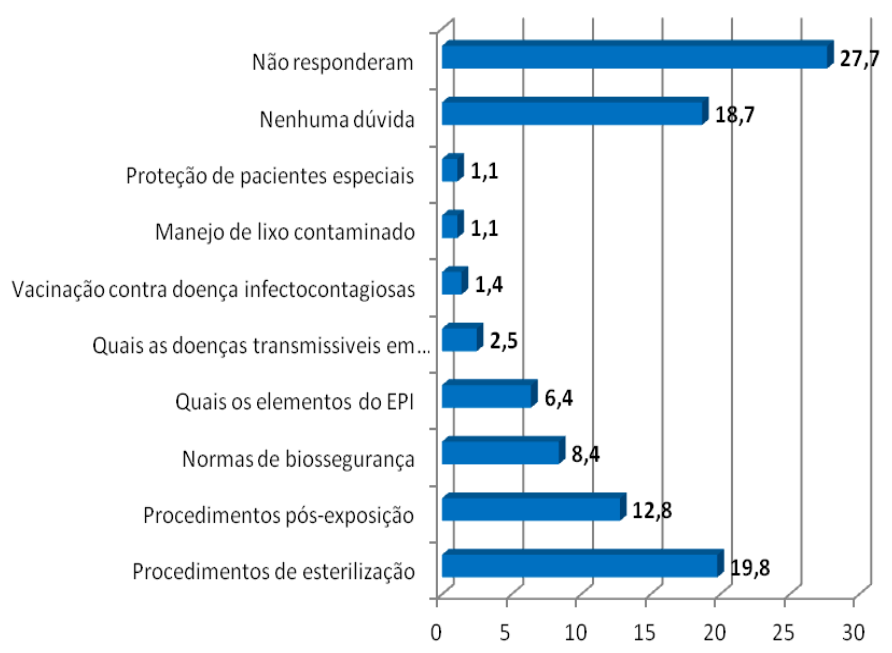

Figura 3. Distribuição das principais dúvidas sobre biossegurança em ambiente odontológico relatadas pela amostra avaliada. Natal - RN, 2012.

Ao quesito "Você acha que todo paciente deve ser tratado como portador de alguma doença?" 288 (80.4\%) alunos responderam afirmativamente e 70 (19.6) responderam que não (Tabela 1).

$\mathrm{Na}$ amostra total, $355(99.2 \%)$ alunos consideraram importante o tema "Biossegurança" para alunos de recém-ingresso ao curso de Odontologia e $3(0.8 \%)$ responderam que não a este quesito (Tabela 1).

\section{Discussão}

A biossegurança objetiva dotar os profissionais e as instituições das medidas e práticas que visem desenvolver as atividades com um grau de segurança adequado, reduzindo ou eliminando os riscos inerentes ao ambiente odontológico. Nesse contexto, é importante, além de reduzir ou eliminar os fatores de risco, avaliar o nível de conhecimento dos futuros profissionais da Odontologia sobre o tema, uma vez que conhecendo o perfil das populações avaliadas, podemse identificar deficiências na adoção de medidas universais de biossegurança, permitindo elaborar programas que visem corrigir as falhas detectadas. Baseado no antes exposto, este estudo pesquisou o conhecimento dos acadêmicos de Odontologia cursando primeiro, quinto e últimos períodos letivos, os quais, certamente podem fornecer dados importantes sobre o conhecimento repassado e adquirido em estágios sensitivos da formação do cirurgião-dentista.

A partir da análise dos dados, foi observado que a grande maioria dos alunos pesquisados conhecia o significado do termo biossegurança, sendo esse grupo formado predominantemente por alunos do quinto e últimos períodos. Como era esperado, a maioria dos alunos que relataram não ter assistido nenhuma aula ou palestra sobre o tema biossegurança e daqueles que não saberiam como se proteger se fossem atender em uma clínica, cursavam o primeiro período. Os conhecimentos sobre biossegurança, a experiência quanto ao uso consciente de EPI e prevenção de acidentes ocupacionais aumentam no decorrer dos períodos cursados (LIMA et al., 2008). Os dados obtidos neste estudo ressaltam a necessidade de se promover um repasse de conhecimentos sobre biossegurança desde o recém-ingresso, visto que é de fundamental importância para o acadêmico e de relevância inestimável para o cirurgião dentista que desde os primeiros períodos do curso de Odontologia seja formada uma sólida base teórica que garanta aos alunos uma entrada às atividades clínicas com maior confiança. De acordo com a atual grade curricular dos cursos avaliados o aluno só terá uma devida instrução sobre o assunto quando encontrar-se no quarto período letivo e prestes a entrar na clínica, não tendo, dessa forma, o conhecimento suficiente e a devida segurança de como se proteger da grande variedade de agentes de risco aos quais estão expostos diariamente na prática odontológica.

Faz-se necessário comentar que alguns alunos do quinto e últimos períodos ao serem questionados também negaram ter tido aula/palestra que focasse o tema biossegurança, sugerindo que, ou esses alunos responderam o questionário sem a atenção adequada, ou ainda, que negligenciaram a importância da pesquisa para a caracterização da amostra em sua instituição, uma vez que, seria inconcebível um aluno que já tem certa prática clínica negue ter tido alguma instrução sobre biossegurança, ou que, realmente sentem-se deficientes no que diz respeito às práticas de proteção, não tendo o conhecimento e ressaltando assim a necessidade de estar sempre se atualizando e reciclando os conhecimentos sobre biossegurança.

Com base nos resultados antes citados, outro fato a ser destacado é a importância do comportamento ético do aluno, o qual precisa ir sendo formado durante todos os períodos do curso, uma vez que, ao serem avaliados com um instrumento como o utilizado neste estudo, ao aceitarem participar voluntariamente, devem estar cientes da responsabilidade ética de fornecer as informações fidedignas que permitirão obter o perfil da população avaliada com o tema pesquisado.

No quesito em que os alunos eram questionados sobre o uso de EPI pelo seu dentista e para o paciente a maioria dos alunos que não notaram o uso de EPI por parte do cirurgião-dentista foram do primeiro período, provavelmente estes resultados estejam associados á falta de conhecimento desses alunos recémingressados sobre os elementos que constituem o EPI em ambiente odontológico. Por outro lado, poucos alunos do quinto e últimos períodos curriculares também relataram não ter percebido o uso de EPI por parte do seu dentista, provavelmente, as respostas desses alunos não refletem a realidade, ou simplesmente foram respostas dadas erroneamente, por falta de atenção com o que estava sendo questionado, uma vez que não se concebe nos dias de hoje um profissional da Odontologia que não utilize pelo menos os elementos básicos do EPI, principalmente, porque sabe-se que o grande número de acidentes ocupacionais está diretamente ligado ao uso incompleto do EPI (RIBEIRO, 2005; MIRANZI, 2003).

Quanto aos EPIs percebidos pelos alunos em seus dentistas, apenas $17.9 \%$ de toda a amostra relatou o uso de EPI completo. Visto que a predominância dos alunos que perceberam a utilização de EPI completo cursava o quinto e últimos períodos e já possuem conhecimento acerca da biossegurança, este dado sugere que os dentistas poderiam estar negligenciando o uso de proteção, ou esses alunos não repassaram nos questionários todos os equipamentos percebidos.

Destacando o papel das universidades na adequada formação teórico-prático-ética dos futuros profissionais da Odontologia, jamais deve-se negligenciar a fiscalização e atualização periódica dos alunos, uma vez que os hábitos e atitudes adquiridos no curso irão nortear a sua futura conduta profissional. Isto pode ser 
exemplificado com os resultados obtidos por Pereira et al. (2005) onde embora os profissionais por eles avaliados tivessem conscientização em relação às medidas de infecção cruzada, estes ainda não praticavam as normas estabelecidas dentro de um rigor profissional, uma vez que foram detectadas falhas em alguns métodos mais primários de proteção individual, como o uso do gorro e do avental de chumbo cervical, sugerindo os autores, a necessidade da implementação de procedimentos e medidas de Biossegurança na prática odontológica e da ampliação dos conhecimentos nesta área, a fim de auxiliar na superação de tais deficiências, visando o controle de infecções e a consequente proteção profissional e dos pacientes.

Devido à manipulação constante de objetos pontiagudos em um local de pequenas proporções, como a boca, os acidentes com material potencialmente contaminante são bastante frequentes na Odontologia (MARZIALE; NISHIMURA; FERREIRA, 2004; CANINI et al., 2002). Com o maior conhecimento sobre doenças como AIDS, hepatite e outras enfermidades que podem ser transmitidas durante um tratamento, as preocupações aumentaram em torno da biossegurança (ANGELO et al., 2007). Os dados deste estudo corroboram o antes citado, uma vez que a grande maioria dos alunos afirmou que trataria todo paciente como portador de alguma doença, reafirmando os achados de outros estudos (PASSOS et al., 2007) . É importante destacar que por diversos motivos, incluindo o medo de não receber atendimento (DISCACCIATI; VILAÇA, 2001), geralmente um paciente não se apresenta ao dentista informando ser portador ou não de doenças, devendo o profissional se resguardar e estar protegido para todos os casos.

Outra preocupação mostrada pelos alunos foi como proceder com a esterilização do material contaminado após um atendimento, destacando o conhecimento dos mesmos com relação a infecções cruzadas. Nesse contexto, resultados da literatura mostram que no Brasil, informações sobre a prevalência de infecções entre os profissionais de saúde ainda são deficientes e os estudos publicados sobre o tema, referem-se à prevalência de infecções entre estudantes universitários, os quais servem como marcadores para infecções relacionadas ao trabalho. Indivíduos com nível universitário lideram o número de acidentes e infecções ocupacionais, seguidos pelos técnicos de laboratório e serventes (SILVA. A.; MASTROENI, 2009).

O protocolo de desinfecção a ser seguido após o atendimento odontológico começa com a eliminação de todo material descartável, devendo todos os materiais pérfuro-cortantes serem dispensados em recipientes apropriados (tipo Descarpack). Seguidamente deve-se colocar o instrumental contaminado em recipientes contendo uma solução de detergente enzimático, por um mínimo de 10 minutos, devendo ser lavados (utilizando-se luvas grossas), secos e acondicionados em caixas metálicas perfuradas e enviados para esterilização em autoclave (SCABELL; JABUR; TINOCO, 2002)

Com relação ao interesse sobre o tema biossegurança, foi observado que $99.2 \%$ dos estudantes consideraram o tema importante, principalmente para os alunos do recém-ingresso, destacando a necessidade de garantir que desde o começo da formação acadêmica seja incentivada a cultura de repasse constante de conhecimentos sobre medidas universais de biossegurança e exposição ocupacional durante as práticas odontológicas.

Nesse contexto é importante citar o relatado por Passos et al de que desde a entrada do acadêmico no curso de Odontologia da Universidade Federal do Ceará, dentre as atividades do Programa de Educação Tutorial (PET) com os alunos recém-ingressados, promovem-se aulas de Introdução à Odontologia dentro do Projeto Recém-ingresso, coordenado pela Pró-Reitoria de Graduação. Tais aulas objetivam dar uma prévia do que esses acadêmicos vão encontrar na faculdade e uma das aulas promovidas pelo PET é sobre biossegurança, o qual constitui um tema de primordial relevância para os profissionais da área de saúde, logo sendo de fundamental importância para a formação do Cirurgião-Dentista.

Acredita-se que o presente estudo possui validade externa, uma vez que a amostra avaliada foi selecionada aleatoriamente e foi representativa das instituições escolhidas para a coleta de dados, mediante a utilização de um instrumento (questionário) previamente testado em outro estudo (PASSOS et al., 2007). Os resultados obtidos, em conjunto e/ou individualmente por cada instituição avaliada, exibiram uma tendência à semelhança, além de corroborarem na sua grande maioria resultados obtidos na literatura, no entanto, considerando que embora existam particularidades das amostras, acredita-se que os resultados deste estudo poderiam ser extrapolados para amostras de outras instituições brasileiras.

\section{Conclusão}

Conclui-se que é importante ministrar conteúdos básicos sobre biossegurança desde os períodos pré-clínicos visando a entrada dos alunos as atividades clínicas com maior segurança, uma vez que o conhecimento prévio e continuado, facilita as tarefas de prevenção de acidentes ocupacionais e o exercício de atividades acadêmicas em segurança em todos os períodos do curso, buscando a proteção tanto do aluno quanto dos pacientes, tendo um papel preponderante nesse processo a atualização e fiscalização constante por parte das equipes docentes.

\section{Referências}

ANGelo, A.R. et al. Pesq. Bras. Odontoped. Clin. Integr., João Pessoa, v.7, n. 3, p. 211-216, set./dez. 2007.

BROZOSKI, M.A. et al. Ocorrência de acidentes pérfuro-cortantes em um curso de odontologia. RGO, Rev. Gaúcha Odontol., Porto Alegre, v. 58, n. 1, p. 77-80, 2010.

CANINI, S.R.M.S. et al. Acidentes perfurocortantes entre trabalhadores de enfermagem de um hospital universitário do interior paulista. Rev. Latino-Am. Enfermagem, Ribeirão Preto, v. 10, n. 2, p. 172-178, mar./abr. 2002.

DISCACCIATI, J.A.C.; VILAÇA, E.L. Atendimento odontológico ao portador do HIV: medo, preconceito e ética profissional. Rev. Panam. Salud Publica $=$ Pan Am. J. Public Health, Washington, v. 9, no. 4, p. 234-239, 2008.

LIMA, A.A. et al. Acidentes ocupacionais: conhecimento, atitudes e experiências de estudantes de odontologia da Universidade Federal da Paraíba. Pesq. Bras. Odontoped. Clín. Integr., João Pessoa, v. 8, n. 3, p. 327-332, set./dez. 2008.

MARZIALE, M.H.P.; NISHIMURA, K.Y.N.; FERREIRA, M.M. Riscos de contaminação ocasionados por acidentes de trabalho com material perfurocortante entre trabalhadores da enfermagem. Rev. LatinoAm. Enfermagem, Ribeirão Preto, v. 12, n. 1, p. 36-42, jan./fev. 2004.

MEDEIROS, U. V.; CARDOSO, A. S.; FERREIRA, S. M. S. Uso das normas de controle de infecção na prática odontológica. Rev. Bras. Odontol., Rio de Janeiro, v. 55, n. 4, p. 209-215, jul./ago. 1998.

MIRANZI, M.A.S. Conhecimentos, atitudes e práticas frente à exposição ocupacional ao HIV entre estudantes, docentes e 
funcionários do curso de odontologia da Universidade de Uberaba. 2003. Tese (Doutorado em Saúde Coletiva) - Faculdade de Ciências Médicas, Universidade Estadual de Campinas, Campinas.

PASSOS, V.F. et al. Biossegurança na visão do recém ingresso no curso de odontologia. [Fortaleza] : Universidade Federal do Ceará, 2007. Disponível em: < http://www.enapet.ufsc.br/anais/BIOSSEGURANCA_NA_VISAO_DO_ RECEM_INGRESSO_NO_CURSO_DE_ODONTOLOGIA.pdf >. Acesso em: 21 jul. $20 \overline{12}$.

PENNA, P.M.M. et al. Biossegurança: uma revisão. Arq. Inst. Biol., São Paulo, v. 77, n. 3, p. 555-565, jul./set. 2010.

PEREIRA, C.V. Avaliação dos conhecimentos dos cirurgiõesdentistas em relação à biossegurança na prática clínica. Rev. Clín. Pesq. Odontol., Curitiba, v. 2, n. 1, p. 19-21, jul./set.

RIBEIRO, P.H.V.; MORIYA, T.M. Acidentes com material biológico potencialmente contaminado em alunos de um curso de odontologia no interior do Paraná. 2005. Dissertação (Mestrado em Enfermagem Fundamental) - Escola de Enfermagem, Universidade Estadual de São Paulo, Ribeirão Preto.

SANTOS, L.F.T.; PELLOGIA, M.C. Conhecimentos, atitudes e comportamento frente aos riscos operacionais dos cirurgiões dentistas do Vale do Paraíba. Rev. Biociênc., Taubaté, v. 8, n. 1, p. 85-93, jan./jun. 2002.

SCABELL, P.L.A.; JABUR, M.S.; TINOCO, B.E.M. Manual de Biossegurança. [Rio de Janeiro]: UERJ, 2002. Disponível em: < http://www.odontologia.uerj.br/fouerj/download/Manuais/bioseguranca .pdf >. Acesso em: 30 jul. 2012.

SILVA, A.D.R.I.; MASTROENI, M.F. Biossegurança: o conhecimento dos formandos da área de saúde. Rev. Baiana, [S.I.], v. 33, n. 3, p. 476-487, jul./set. 2009.

SILVA, G.S. et al. Conhecimento e utilização de medidas de precaução-padrão por profissionais de saúde. Esc. Anna Nery, [S.I.], v. 16, n.1, p. 103-110, mar. 2012.

SILVA, R. H. B. T. et al. Levantamento dos métodos de controle de infecção cruzada utilizados pelos cirurgiões-dentistas, auxiliares e estudantes de odontologia do município de Araraquara-SP. RFO UPF, Passo Fundo, v. 12, n. 2, p. 7-12, maio/ago. 2007.

Rev. Fac. Odontol. Porto Alegre, v. 53, n. 1, p. 11-15, jan./abr., 2012. 